\title{
Correspondence
}

\section{Ezetimibe, Oxidized Low Density Lipoprotein, Lp (a), and Dyslipidemia}

\author{
Henrique A. R. Fonseca, Maria C. Izar, Henrique T. Bianco, and Francisco A. Fonseca
}

Cardiology Division, Department of Medicine, Federal University of São Paulo, São Paulo, S.P., Brazil

In the recent paper published by Nozue et al. ( Atheroscler Thromb, 2010; 17(1): 37-44) ${ }^{1)}$, the authors investigated the effects of the intestinal cholesterol absorption inhibitor, ezetimibe, on lipids and other risk markers for atherosclerosis in subjects with hyperlipidemia.

The effects of ezetimibe in different clinical scenarios of cardiovascular disease are not well known, and its effects beyond cholesterol reduction are yet less explored, when compared with those attributed to the inhibition of cholesterol synthesis by statins. In the paper published by Nozue et al., the authors indicated that the administration of ezetimibe for 12 weeks resulted in beneficial effects on biomarkers for atherosclerosis.

Nozue et al. postulated that the two groups, ezetimibe $10 \mathrm{mg}$ monotherapy, and ezetimibe $10 \mathrm{mg}$ plus a statin, apparently produced the same results after 12-week follow-up; however, there was no study regarding the effects of statins alone, since these agents are capable of reducing low-density lipoprotein cholesterol (LDL-C), C-reactive protein (hs-CRP), triglycerides, and oxidized LDL (oxLDL) $\left.{ }^{2}, 3\right)$; in addition, the particular potency of each drug as a hypolipidemic agent could have been taken into account, as demonstrated in the Discovery study ${ }^{4)}$.

These observations on lipid alterations and oxidative parameters could have been evaluated with the addition of another group of patients receiving a statin alone in a dose equivalent to that of a statin combined with ezetimibe, of the same class or with similar hypolipidemic potency. We observed that in the group that received statins there were patients with type 2 diabetes and a history of coronary artery disease, compared with the group without these metabolic and inflammatory alterations. It is already known that in this patient population the response to hypolipidemic strategies may differ substantially, as diabetic patients have

Address for correspondence: Maria Cristina de Oliveira Izar, Federal University of São Paulo, Rua Pedro de Toledo, 276, Vila Clementino, São Paulo, S.P., 04039030, Brazil

E-mail:mcoizar@terra.com.br increased cholesterol absorption.

The results were presented by grouping the two treatment arms and were quoted as the general effects of ezetimibe, an issue that can affect the findings, since it does not control for other variables, beyond statin effects, such as diet, intensity and volume of exercise performed by the study patients. These interventions can also alter triglycerides and oxLDL concentrations ${ }^{5)}$.

The conclusion that ezetimibe can reduce markers of atherosclerosis and be a potential therapeutic agent for the reduction of lipoprotein (a), and to modify other markers still needs to be confirmed in larger studies addressing its direct effect or the effect of the combination of hypolipidemic therapies.

\section{References}

1) Nozue T, Michishita I, Mizuguchi I: Effects of ezetimibe on remnant-like particle cholesterol, lipoprotein (a), and oxidized low-density lipoprotein in patients with dyslipidemia. J Atheroscler Thromb, 2010; 17: 37-44

2) Choi SH, Chae A, Miller E, Messig M, Ntanios F, DeMaria AN, Nissen SE, Witztum JL, Tsimikas S: Relationship between biomarkers of oxidized low-density lipoprotein, statin therapy, quantitative coronary angiography, and atheroma: volume observations from the REVERSAL (Reversal of Atherosclerosis with Aggressive Lipid Lowering) study. J Am Coll Cardiol, 2008; 52: 24-32

3) Ridker PM, Danielson E, Fonseca FA, Genest J, Gotto AM Jr, Kastelein JJ, Koenig W, Libby P, Lorenzatti AJ, Macfadyen JG, Nordestgaard BG, Shepherd J, Willerson JT, Glynn RJ; JUPITER Trial Study Group: Reduction in C-reactive protein and LDL cholesterol and cardiovascular event rates after initiation of rosuvastatin: a prospective study of the JUPITER trial. Lancet, 2009; 373: 1175 1182

4) Middleton A, Binbrek AS, Fonseca FA, Wilpshaar W, Watkins C, Strandberg TE: Achieving 2003 European lipid goals with rosuvastatin and comparator statins in 6743 patients in real-life clinical practice: DISCOVERY meta-analysis. Curr Med Res Opin, 2006; (6): 1181-1191

5) Cornelissen VA, Arnout J, Holvoet P, Fagard RH: Influence of exercise at lower and higher intensity on blood pressure and cardiovascular risk factors at older age. J Hypertens, 2009; 27: 753-762 